\title{
FF-Anonymity: When Quasi-Identifiers Are Missing
}

\author{
Ke Wang ${ }^{\# 1}$, Yabo $\mathrm{Xu}{ }^{\# 1}$, Ada W.C. Fu ${ }^{* 2}$, Raymond C.W. Wong ${ }^{* 2}$ \\ \# Simon Fraser University \\ 1 \{wangk,yxu\}@cs.sfu.ca \\ *The Chinese University of Hong Kong \\ ${ }^{2}$ \{adafu, cwwong\}@cse. cuhk. edu.hk
}

\begin{abstract}
Existing approaches on privacy-preserving data publishing rely on the assumption that data can be divided into quasi-identifier attributes (QI) and sensitive attribute (SA). This assumption does not hold when an attribute has both sensitive values and identifying values, which is typically the case. In this paper, we study how such attributes would impact the privacy model and data anonymization. We identify a new form of attacks, called "freeform attacks", that occur on such data without explicit QI attributes and SA attributes. We present a framework for modeling identifying/sensitive information at the value level, define a problem to eliminate freeform attacks, and outline an efficient solution.
\end{abstract}

\section{INTRODUCTION}

Privacy-preserving data publishing (PPDP) focuses on publishing person-specific data (also called microdata) for the benefit of research. Previous works have considered microdata of the form $T\left(A_{1}, \ldots, A_{m}, S A\right)$. Each record corresponds to an individual. $\mathrm{SA}$ is the sensitive attribute. $\mathrm{QI}=\left\{A_{1}, \cdots, A_{m}\right\}$, called the quasi-identifier, is a set of attributes that can be linked with an external source such as a voter list. In a linking attack, the adversary knows that some individual has a record in $T$, and observes the information $q i$ on QI about the individual from an external source. The adversary's goal is to find the SA value of the individual. If the records in $T$ that match $q i$ are predominantly associated with a common SA value, the adversary could infer the individual's SA value with a high probability.

\section{A. Abandoning the QI/SA paradigm}

A fundamental assumption in previous works is that $T$ can be split (vertically) into QI and the sensitive attribute SA. In this paper, the term QI/SA paradigm refers to this assumption and the works based on it. $k$-anonymity [1], $l$-diversity [2] and recent works all fall into this QI/SA paradigm. A common approach in the QI/SA paradigm is hiding the association between QI and SA. To this end, the generalization approach [1] partitions records into equivalence classes according to QI, and the bucketization approach [3] split records into sub-records according to the partition of QI and SA. Our insight is that determining QI and SA can be tricky and even undesirable. To explain this point, let us consider an example.

Example 1.1: Consider the table T1 on (Sex, Income, Disease) in Figure 1(a). Income and Disease have a hierarchical domain given by the taxonomies on the top of the figure. In our discussion, we use VI for "Viral Infectious", BI for

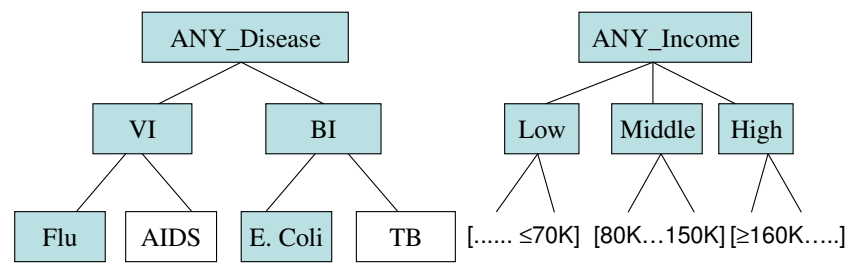

\begin{tabular}{|l|l|l|}
\hline Sex & Income & Disease \\
\hline F & $190 \mathrm{~K}$ & Flu \\
\hline M & $190 \mathrm{~K}$ & AIDS \\
\hline F & $160 \mathrm{~K}$ & AIDS \\
\hline M & $160 \mathrm{~K}$ & AIDS \\
\hline M & $100 \mathrm{~K}$ & E. Coli \\
\hline M & $80 \mathrm{~K}$ & E. Coli \\
\hline F & $80 \mathrm{~K}$ & TB \\
\hline F & $100 \mathrm{~K}$ & TB \\
\hline \multicolumn{3}{|c|}{ (a) T1: Raw table } \\
\hline \multicolumn{3}{|c}{}
\end{tabular}
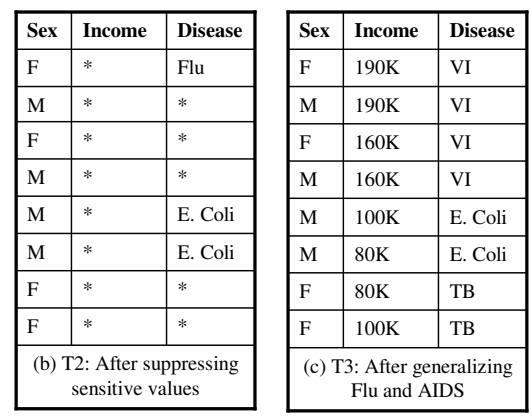

Fig. 1. A motivating example

"Bacterial Infectious", E.Coli for "Escherichia coli", and TB for "Tubercle bacillus". Suppose that the following holds:

A1 Disease: Flu and E.Coli are non-sensitive and can be observed on an individual; AIDS and TB are sensitive and cannot be observed.

A2 Income: an exact income is sensitive and cannot be observed, but an income bracket such as High, Middle, and Low is non-sensitive and can be observed.

A3 Sex: $F$ and $M$ are non-sensitive and can be observed.

We also assume that, if a value is non-sensitive, so are more generalized values. In a taxonomy, a non-sensitive value is denoted by a shaded node. The terms "non-sensitive", "observable" and "public" are interchangeable.

Within the QI/SA paradigm, since Income and Disease contain sensitive values, they can only be modeled as the sensitive attribute SA. The following are two possible ways of modeling:

- Case 1: $Q I=\{S e x\}$ and $S A=$ Disease, and

- Case 2: $Q I=\{\operatorname{Sex}\}$ and SA=Income.

Suppose that we consider 2-diversity [2] as our privacy goal. It is easy to see that T1 satisfies 2-diversity in both Case 1 and Case 2: no single SA value occurs in more than 1/2 percent 
of the records sharing a common value on QI. Therefore, the probability of linking an individual to SA via QI is no more than $50 \%$.

However, publishing $T 1$ is not safe under our assumptions A1, A2 and A3. Consider an adversary who tries to find the individual's sensitive value. First, with $M$ and High being observable (i.e., A2 and A3), the adversary can link an individual $P_{1}$ via $M$ and High to the two records $(M, 190 K, A I D S)$ and $(M, 160 K, A I D S)$ and infer AIDS with $100 \%$ certainty. Note that this linking does not require observing the exact income of the individual. Let us denote this attack by

$$
\{M, H i g h\} \rightarrow A I D S \text {. }
$$

Second, with F and Flu being observable (i.e., Al and A3), the adversary can link an individual $P_{2}$ to the first record via $F$ and Flu, thus, to the exact income 190K, with $100 \%$ certainty. Let us denote this attack by

$$
\{F, F l u\} \rightarrow 190 K \text {. }
$$

These attacks exist even though $T 1$ is 2-diverse in both Case 1 and Case 2. Note that in these attacks, Disease acts as both an identifying attribute (in $\{F, F l u\} \rightarrow 190 K$ ) and $S A$ (in $\{M, H i g h\} \rightarrow A I D S)$. To prevent such attacks, new solutions are required.

One solution is suppressing the sensitive AIDS, TB and all exact incomes, as in T2 in Figure 1(b). This solution loses too much information since sensitive values usually are important for data analysis. A preferred solution is generalizing Flu and AIDS to VI, as in Figure 1(c), and the result table T3 is safe for release. For example, $\{F, V I\} \rightarrow 190 \mathrm{~K}$ has only $50 \%$ certainty and $\{M, H i g h\} \rightarrow V I$ is no longer a threat because VI is non-sensitive.

This example illustrates several interesting points.

First, information on sensitive attributes may be observed on an individual. The QI/SA paradigm assumes that no information on SA can be observed on an individual. In the above example, Flu for Disease and High for Income can be observed. Both Disease and Income are considered sensitive attributes. This example shows that, even for such attributes, a less sensitive value or a higher level value can be easily observed.

Second, "public" and "sensitive" information often is distinguished at the value level. The QI/SA paradigm assumes that for each attribute, either all values are public or all values are sensitive. In the above example, Income and Disease have both types of values. The QI/SA paradigm is handicapped in dealing with such attributes: treating them as SA would under-protect the data because observable values (such as Flu and $H i g h)$ ) are mistakenly treated as non-observable, whereas treating them as QI attributes would over-protect because some sensitive values (such as $190 \mathrm{~K}$ and $A I D S$ ) may not be easily observed.

Third, a linking attack may be through observing a different set of attributes for a different individual. The attack $\{M, H i g h\} \rightarrow A I D S$ on the individual $P_{1}$ is by observing values on Sex and Income, whereas the attack $\{F, F l u\} \rightarrow$ $190 \mathrm{~K}$ on the individual $P_{2}$ is by observing values on Sex and Disease. The QI/SA paradigm essentially assumes that all individuals have the same set of observable attributes and the same sensitive attribute. This is not reasonable because the adversary will not confine herself to any pre-determined QI.

\section{B. Challenges and contributions}

We consider a table $T\left(A_{1}, \ldots, A_{m}\right)$ where each attribute $A_{i}$ has both observable values and sensitive values. To our knowledge, this is the first work that addresses the linking attacks under this setting. The following summarizes the challenges and our contributions.

Challenge/Contribution 1: The first challenge is modeling observability and sensitivity "at the value level". Requiring the publisher to specify this information for all domain and generalized values is neither feasible nor scalable. We present a framework for these specifications, with an focus on general principles so that new instantiations can be adapted.

Challenge/Contribution 2: We identify a class of freeform attacks of the form $X \rightarrow a$, where $a$ and the values in $X$ can be at any level of any attributes. $X \rightarrow a$ is a privacy breach if $X$ is observable, $a$ is sensitive, and $X$ is associated with $a$. As shown in Example 1.1, a unique challenge posed by freeform attacks is that they may occur at a general level without occurring at a special level. We propose the notion of $F F$-anonymity ( $\underline{F}$ ree $\underline{F}$ orm-anonymity) to eliminate freeform attacks.

Challenge/Contribution 3: We show that finding an optimal FF-anonymization is NP-hard and present an efficient solution for finding a "minimally generalized", not necessarily optimal, FF-anonymization.

\section{Modeling Sensitivity And ObSeRvability}

\section{A. Terminology}

Consider a set of attributes $U=\left\{A_{1}, \ldots, A_{m}\right\}$. Each $A_{j}$ has a taxonomy (tree) arranged from general values at high levels to specific values at low levels. "Nodes" and "values" are interchangeable. $A N Y_{j}$ denotes the root of the taxonomy for $A_{j}, \operatorname{Subtr}(a)$ denotes the subtree under a node $a$, and Leaf $(a)$ denotes the set of all leaf nodes in $\operatorname{Subtr}(a)$. For two values $a$ and $a^{\prime}, a^{\prime} \succeq a$ means that either $a^{\prime}=a$ or $a^{\prime}$ is an ancestor of $a$.

A base table over $U$, denoted $T$, contains only leaf values. A generalized table over $U$, denoted $T^{*}$, is produced by applying zero or more generalization to $T$. Each generalization replaces all child values $v_{1}, \cdots, v_{p}$ with their parent $v$ in all records containing $v_{1}, \cdots, v_{p} . T^{*}$ can also be produced by applying a sequence of specializations to $T^{\top}$, where $T^{\top}$ denotes the most generalized table containing $A N Y_{i}$ for every attribute $A_{i}$. Each specialization replaces a parent value $v$ in every record containing $v$ with the child value that generalizes the original value in the record. For two generalized tables $T_{2}^{*}$ and $T_{1}^{*}$, we say that $T_{2}^{*}$ is more general than $T_{1}^{*}$ if $T_{2}^{*}$ is obtained from $T_{1}^{*}$ by zero or more generalization.

Let $C u t\left(A_{i}, T^{*}\right)$ denote the set of values in $T^{*}$ on $A_{i}$, and $\mathrm{Cut}^{+}\left(A_{i}, T^{*}\right)$ denote the set of values in $\operatorname{Cut}\left(A_{i}, T^{*}\right)$ plus all ancestors. For a set of attributes Attset, $\mathrm{Cut}^{+}\left(\right.$Attset, $\left.T^{*}\right)$ denotes the cross product $\times_{A_{i} \in \text { Attset }} C u t^{+}\left(A_{i}, T^{*}\right)$. A valueset 
in $\mathrm{Cut}^{+}\left(\right.$Attset, $\left.T^{*}\right)$ is a tuple containing exactly one value from $\mathrm{Cut}^{+}\left(A_{i}, T^{*}\right)$ for each attribute $A_{i}$ in Attset. For two valuesets $X$ and $X^{\prime}$ in $C_{u t}^{+}\left(\right.$Attset, $\left.T^{*}\right), X^{\prime} \succeq X$ means that for every $A_{i}$ in Attset, $X^{\prime}\left[A_{i}\right] \succeq X\left[A_{i}\right]$, where $X\left[A_{i}\right]$ denotes the value in $X$ on $A_{i}$. For a record $r$ in $T^{*}$, we say that $r$ matches $X$ if $X \succeq r[$ Attset $]$. $\sup (X)$ denotes the number of records in $T^{*}$ that match $X$.

\section{B. Sensitivity model}

Consider a value $a$ for some attribute $A_{i}$. The sensitivity of $a$, denoted $s(a)$, measures the degree to which the publisher considers $a$ sensitive. We assume that the publisher is able to specify each leaf node $a$ as either sensitive or non-sensitive, i.e., $s(a)=1$ or $s(a)=0$, respectively. This can be done by specifying a set of highest possible nodes to cover all sensitive leaf nodes, called a guarding set. A leaf node $a$ is sensitive if and only if $a$ is in $\operatorname{Leaf}(g)$ for some node $g$ in the guarding set. Let $s L e a f(a)$ denote the set of sensitive leaf nodes in Leaf $(a)$.

Example 2.1: Consider the taxonomy for Disease in Figure 1. The guarding set $G S 1=\{A I D S, T B\}$ specifies the sensitive leaf nodes $A I D S$ and TB. GS2 $=\{A I D S, B I\}$ specifies the sensitive leaf nodes AIDS, E.Coli and TB.

The publisher does not need to specify $s(a)$ for nonleaf nodes $a$ because this will be specified automatically by our model. For a non-leaf node $a, s(a)$ depends on what $a$ generalizes, i.e., the leaf nodes in Leaf $(a)$. For instance, $A N Y_{-}$Disease is more sensitive if most leaf nodes in Leaf $\left(A N Y \_\right.$Disease $)$are sensitive. This motivates the following principle.

Definition 2.1 (Sensitivity Principle): The sensitivity of a, $s(a)$, conveys the probability that a originates from sensitive leaf values in Leaf $(a)$. This probability is the publisher's interpretation of the sensitivity of $a$.

The exact interpretation of probability depends on the instantiation for " $a$ originates from sensitive leaf values in Leaf $(a)$ ". We leave this instantiation open so that a new instantiation can be "plugged in" through $s(a)$. Below, we demonstrate this flexibility by considering two instantiations.

The aggregate instantiation. In this instantiation, $s(a)$ measures the probability that $a$ comes from any sensitive leaf node in Leaf $(a)$, defined as:

$$
s(a)=|s \operatorname{Leaf}(a)| /|\operatorname{Leaf}(a)| .
$$

That is, $s(a)$ is the fraction of sensitive leaf nodes in Leaf $(a)$. In particular, this instantiation makes no distinction among individual sensitive leaf nodes and the publisher considers it sensitive to infer that $a$ comes from any sensitive leaf node $\operatorname{sLeaf}(a)$.

Example 2.2: Continue with the guarding set $G S 1=$ $\{A I D S, T B\} . A I D S$ and $T B$ are sensitive leaf nodes and Flu and E.Coli are non-sensitive leaf nodes. $s(A I D S)=$ $s(T B)=1, s(F l u)=s($ E.Coli $)=0 . s(a)=1 / 2$ for $V I, B I, A N Y$ _Disease. For example, s $\left(A N Y_{-}\right.$Disease $)=$ $1 / 2$ because $50 \%$ of leaf nodes are sensitive.
The non-aggregate instantiation. In many cases, sensitivity arises from the specificity of a property. Location privacy is such an example. This notion of sensitivity $s(a)$ can be measured by the probability that $a$ comes from a particular sensitive leaf node in Leaf $(a)$. Unlike the aggregate instantiation, this instantiation distinguishes among sensitive leaf nodes. Under the uniform distribution, the probability that $a$ comes from a particular leaf node in Leaf $(a)$ is $1 / \mid$ Leaf $(a) \mid$ and the probability that a given leaf node in Leaf $(a)$ is sensitive is $\mid$ sLeaf $(a)|/| \operatorname{Lea} f(a) \mid$. Therefore, under the independence assumption, the probability that $a$ comes from a particular sensitive leaf node is

$$
s(a)=|s \operatorname{Leaf}(a)| /|\operatorname{Leaf}(a)|^{2} .
$$

There is no requirement that the same instantiation be used for all attributes. The choice of instantiation should be based on what probabilistic interpretation is desired for an attribute.

\section{Observability model}

For a valueset $X$ in $\mathrm{Cut}^{+}\left(\right.$Attset, $\left.T^{*}\right)$, the observability of $X$, denoted $o(X)$, measures the degree to which the publisher considers $X$ as observable to the adversary. Our observation is that, for two valuesets $X$ and $X^{\prime}$ with $X^{\prime} \succeq X$, whenever $X$ is observed on some individual, $X^{\prime}$ is observed on the same individual because $X$ is an instance of $X^{\prime}$. For example, observing the exact income $170 \mathrm{~K}$ implies observing the income bracket High. This observation leads to the following principle.

Definition 2.2 (Observability Principle): The observability of a valueset $X$, denoted $o(X)$, conveys some notion of the degree that $X$ may be observed on an individual with the following property: for $X^{\prime} \succeq X, o\left(X^{\prime}\right) \geq o(X)$; in other words, a general valueset is as observed as a special one.

Since observing a valueset $X$ entails observing every value in $X$, the "bottleneck" is the least observed value in $X$. So we define $o(X)$ by $\min \{o(a) \mid a \in X\}$. For a given threshold $\sigma_{o}$, we say that $X$ is observable if $o(X) \geq \sigma_{o}$. Below, we consider two instantiations for specifying $o(a)$ for a single value $a$ on some attribute.

The sensitivity based instantiation. The first instantiation is based on the intuition that a sensitive value is hard to observe and a non-sensitive value is easy to observe. Therefore, if all the leaf nodes in Leaf $(a)$ are sensitive, it is hard to observe $a$, so $o(a)=0$; if some leaf node in Leaf $(a)$ is non-sensitive, such leaf nodes can be observed, and $a$ can be observed following Observability Principle, so $o(a)=1$.

Example 2.3: Continue with the guarding set $G S 1=$ $\{A I D S, T B\}$ in Example 2.1. $o(A I D S)=o(T B)=$ 0 because these nodes are sensitive. $o(a)=1$ for Flu, E.Coli, VI, BI, ANY_Disease because these nodes have some non-sensitive leaf nodes.

The belief based instantiation. Sometimes the publisher wants to specify $o(a)$, in the range [0..1], according to her own belief. Suppose that the publisher has specified $o(a)$ for all leaf nodes $a$. For a non-leaf node $a$, Observability Principle requires $o(a) \geq o\left(a_{i}\right)$ for all child nodes $a_{i}$ of $a$. A valid 
specification is $o(a)=\min \left\{1, \alpha+\max _{i} o\left(a_{i}\right)\right\}$, where $\alpha$ is a "boost" of observability when going from the child level to the parent. For example, $o\left(a_{i}\right)=0$ and $\alpha=1$ specifies that exact incomes $a_{i}$ cannot be observed but the income bracket $a$ can be observed. However, the specification $o(a)=a v g_{i} o\left(a_{i}\right)$ violates Observability Principle because $\operatorname{avg}_{i} o\left(a_{i}\right)$ may be smaller than $o\left(a_{i}\right)$.

Corollary 2.1: The sensitivity based and belief based instantiations satisfy Observability Principle.

Our approach does not depend on the exact instantiation for $o(X)$ and $s(a)$. The only requirement is that they follow Observability Principle and Sensitivity Principle. Therefore, a new instantiation for $o(X)$ and $s(a)$ can be easily incorporated into our approach.

\section{Problem Statements}

\section{A. Privacy breaches}

Consider a published table $T^{*}$. The adversary attempts to infer some value $a$ in $C u t\left(A, T^{*}\right)$ for some attribute $A$ by observing a valueset $X$ in $C u t^{+}\left(U-A, T^{*}\right)$. In the following discussion, we assume that $X$ is observable (otherwise, $X$ cannot be linked to any individual) and $a$ is non-observable (otherwise, there is no need to infer $a$ ).

Definition 3.1 (Attacks): Given a threshold $\sigma_{o}$ on observability, $a$ freeform attack wrt $T^{*}$ has the form $X \rightarrow a$, where $a$ is a non-observable value in $C u t\left(A, T^{*}\right)$ for some attribute $A$ and $X$ is an observable valueset in $C^{+} t^{+}\left(U-A, T^{*}\right)$.

Definition 3.2 (Threat): Given $T^{*}$ and the taxonomies for all attributes, the threat of $X \rightarrow a$ wrt $T^{*}$, denoted $t(X \rightarrow$ $a)$, is the probability with which the adversary can infer that a record in $T^{*}$ matching $X$ "originates" from sensitive leaf nodes in Leaf $(a)$.

$t(X \rightarrow a)$ depends on two probabilities. The first is the probability that a record in $T^{*}$ matching $X$ matches $a$, i.e., $P(a \mid X)=\frac{\sup (X a)}{\sup (X)}$. The second is the probability that $a$ originates from a sensitive leaf node in Leaf $(a)$, i.e., $s(a)$.

Theorem 3.1: For an attack $X \rightarrow a$ wrt $T^{*}$, under the independence assumption of $P(a \mid X)$ and $s(a)$,

$$
t(X \rightarrow a)=P(a \mid X) * s(a) .
$$

With the aggregate instantiation of $s(a), t(X \rightarrow a)$ is the probability that a record in $T^{*}$ matching $X$ is associated with any sensitive leaf node in $\operatorname{Leaf}(a)$; with the non-aggregate instantiation of $s(a), t(X \rightarrow a)$ is the probability that a record in $T^{*}$ matching $X$ is associated with a particular sensitive leaf node in $\operatorname{Leaf}(a)$. We consider $X \rightarrow a$ to be a privacy breach if $t(X \rightarrow a)$ is large enough.

Definition 3.3 (Breaches): Given thresholds $\sigma_{o}$ and $\sigma_{t}$ on observability and threat, any attack $X \rightarrow a$ wrt $T^{*}$ is said to be a breach if $t(X \rightarrow a)>\sigma_{t} . B R\left(T^{*}\right)$ denotes the set of all breaches wrt $T^{*}$.

We are interested in finding a minimally generalized $T^{*}$ with $B R\left(T^{*}\right)=\emptyset$. The difficulty is that $B R\left(T^{*}\right)$ is not monotone wrt generalization: $B R\left(T_{2}^{*}\right)$ is not necessarily a subset of $B R\left(T_{1}^{*}\right)$, where $T_{2}^{*}$ is more general than $T_{1}^{*}$, because a generalization may increase the threat of an attack.
Consider generalizing leaf nodes $a_{1}$ and $a_{2}$ into the parent $a$, where $s\left(a_{1}\right)=1$ and $s\left(a_{2}\right)=0$. Suppose that, before the generalization, all matching records of $X$ have $a_{2}$. So $t\left(X \rightarrow a_{1}\right)=t\left(X \rightarrow a_{2}\right)=0$. After the generalization, $t(X \rightarrow a)=1 / 2$ because $s(a)=1 / 2$ (assuming the aggregate instantiation) and $P(a \mid X)=P\left(a_{2} \mid X\right)=1$. To address this issue, we extend $B R\left(T^{*}\right)$ to $X \rightarrow a$, where $a$ can be more general than the values in $T^{*}$. This is defined below.

Definition 3.4 (Extended Breaches): Any attack $X \rightarrow$ a wrt $T^{*}$, with a being to a non-observable value in $\mathrm{Cut}^{+}\left(A, T^{*}\right)$ for some attribute $A$, is said to be a breach if $t(X \rightarrow a)>\sigma_{t}$. $B R\left(T^{*}\right)$ denotes the set of all breaches wrt $T^{*}$.

Note the difference between Definition 3.3 and 3.4: the latter includes those $X \rightarrow a$ with $a$ being more general than the values in $T^{*}$. With this extended $B R\left(T^{*}\right)$, it follows from the definition that, if $T_{2}^{*}$ is more general than $T_{1}^{*}, B R\left(T_{2}^{*}\right)$ is a subset of $B R\left(T_{1}^{*}\right)$.

Corollary 3.1: For $B R\left(T^{*}\right)$ in Definition 3.4, if $T_{2}^{*}$ is more general than $T_{1}^{*}, B R\left(T_{2}^{*}\right) \subseteq B R\left(T_{1}^{*}\right)$.

Several remarks are in place. First, by considering the extended $B R\left(T^{*}\right)$, Corollary 3.1 allows a top-down search for a maximally specialized $T^{*}$ with $B R\left(T^{*}\right)=\emptyset$ : starting with the most generalized table $T^{*}=T^{\top}$, we iteratively specialize it on some attribute as long as $B R\left(T^{*}\right)=\emptyset$. Second, the extended $B R\left(T^{*}\right)$ is a superset of the breaches defined in Definition 3.3; therefore, $B R\left(T^{*}\right)=\emptyset$ is a sufficient condition for eliminating all the breaches in Definition 3.3. In the rest of the paper, $B R\left(T^{*}\right)$ refers to the extended $B R\left(T^{*}\right)$ in Definition 3.4. Finally, Corollary 3.1 does not depend on the instantiation of $s(a)$, therefore, the top-down approach remains applicable if a new instantiation of $s(a)$ is adapted.

\section{B. Anonymization problems}

Let $I L\left(T^{*}, T\right)$ denote a chosen metric for information loss of generalizing $T$ to $T^{*}$.

Definition 3.5: We say that $T^{*}$ is an $\mathrm{FF}$-anonymization of $T$ if $B R\left(T^{*}\right)=\emptyset$. Problem I: find an optimal FF-anonymization $T^{*}$, that is, for every FF-anonymization $T_{2}^{*}, I L\left(T^{*}, T\right) \leq$ $I L\left(T_{2}^{*}, T\right)$. Problem II: find a minimal FF-anonymization $T^{*}$, that is, no specialization on $T^{*}$ leads to an FF-anonymization.

We can show that Problem I is NP-hard by a reduction from the optimal $k$-anonymity problem to Problem I. With Corollary 3.1, we can find a solution to Problem II, i.e., a minimal FFanonymization, following a top-down specialization process starting with the most generalized table $T^{*}=T^{\top}$. Corollary 3.1 implies that further specialization leads to no solution. The detailed algorithm and evaluation will be presented in the full version of this paper.

\section{REFERENCES}

[1] L. Sweeney, " $k$-anonymity: A model for protecting privacy," International Journal on Uncertainty, Fuzziness and Knowledge-based Systems, vol. 10, no. 5, 2002.

[2] A. Machanavajjhala, J. Gehrke, D. Kifer, and M. Venkitasubramaniam, "l-diversity: Privacy beyond k-anonymity," in ICDE, Atlanta, GA, 2006.

[3] X. Xiao and Y. Tao, "Anatomy: Simple and effective privacy preservation," in Proc. of the 32nd International Conference on Very Large Data Bases (VLDB), Seoul, Korea, September 2006. 